\title{
Revisión sobre los usos y variables para determinar la calidad del agua pluvial en superficies de cubierta
}

pags $146-159$

Grupo de Investigación: territorio y habitabilidad

Línea de investigación: Territorio, ciudad y ambiente

Oscar Cortés Cely•

\section{RESUMEN}

El artículo profundiza sobre los usos, variables y superficies de cubierta que garantizan la calidad del agua lluvia, los sistemas de captación y la tecnología vigente sobre el tema. Se analizan 50 artículos relacionados con la investigación, siendo Asia y Norte América las regiones con el mayor número de publicaciones científicas. La mayor parte de los artículos están relacionados con el uso final que se da al recurso pluvial en el mundo, en segundo lugar los artículos publicados analizan las variables del agua lluvia para su almacenaje y potencial uso. $Y$ finalmente en menor producción las publicaciones relacionadas sobre las superficies de captación en diferentes tipos y materiales de cubierta.

Palabras clave: Agua pluvial, Calidad agua pluvial, Superficies de cubierta.

\section{ABSTRACT}

The article elaborates on the uses, variables and roof surfaces that guarantee the quality of rain water catchment systems and current technology on the subject. 50 articles related to the research are analyzed, with Asia and North America regions with the largest number of scientific publications. Most items are related to the end-use resource to rain occurs in the world, second published articles analyzing the variables rainwater for storage and potential use. And finally in lower production publications they are related to the collection surfaces in different types and cover materials.

Key words: Rainwater, Storm water quality, Indoor surfaces. - Arquitecto especialista en educación artística y master en arquitectura bioclimática, docente de la facultad de Arquitectura e
Investigador de la Fundación Universidad de América. oscar.cortes@profesores.uamerica.edu.co. 
LINNEA DE INVESTIGACIÓN: ARQUITECTURA, CIUDAD Y AMBIENTE

\section{INTRODUCCIÓN}

Una de las consecuencias del calentamiento global y de las variaciones de los patrones de lluvia es el crecimiento y densificación de los centros urbanos de las grandes ciudades en el mundo, el cambio de las dinámicas sociales, culturales y económicas han convertido a la ciudad en el "lugar" de preferencia de las personas y de las familias que se han desplazado del campo y de las regiones más apartadas a las grandes ciudades del mundo entero. La demanda de agua ejerce presión sobre el recurso hídrico, se requiere por lo tanto pensar en otras alternativas de captación, gestión y utilización de este importante recurso para la supervivencia humana. Países como Alemania, Australia y Canadá, vienen implementando la captación de agua lluvia como una alternativa de sostenibilidad ambiental (Despins et al, 2009). La principal preocupación a nivel mundial es la calidad del agua pluvial captada, su almacenamiento y distribución, considerando que el principal objetivo es el uso doméstico y como usos secundarios; la limpieza y mantenimiento de fachadas, descarga del inodoro, el lavado de autos y riego de plantas (Woltersdorf et al, 2013).

La recolección de agua lluvia es una fuente alternativa de agua frente al cambio climático y déficit de agua potable en el mundo (Steffen et al, 2013). Las superficies de cubiertas conforman un importante volumen del recurso hídrico pluvial cuando se presentan temporadas de lluvia que permiten su captación y aprovechamiento, necesariamente se requiere determinar la calidad de agua pluvial, según el uso y las necesidades de los usuarios en las grandes ciudades por déficit en la demanda y en pequeñas comunidades por la dificultad del abastecimiento o escases del recurso. En la presente revisión se tuvieron en cuenta tres tópicos fundamentales que hacen parte del análisis de la investigación; el primer tópico hace referencia al uso final del agua pluvial, es decir el destino para el cual se va a emplear, el segundo tópico contempla las variables que determinan la calidad del agua pluvial y por último el análisis del tipo de super- ficies de cubierta que se emplean para mejorar las condiciones de captación y calidad del agua pluvial.

\section{REVISIÓN CIENTÍFICA}

En la revisión científica se incluyeron artículos encontrados en revistas indexadas internacionalmente, a través de bases de datos como Ebsco Host, ISI, y Scopus, el $90 \%$ de los artículos consultados están publicados en inglés, dentro del análisis de la información se revisaron 50 artículos publicados entre 2005 y 2014 de los cuales el $58 \%$ corresponden al principal tema de investigación; usos del agua pluvial; 29 artículos revisados, en segundo lugar los artículos referentes a la calidad del agua; se revisaron 27 artículos que corresponden al $54 \%$ de las publicaciones indexadas y el tercer tópico referente a las superficies de cubierta; se revisaron 13 artículos que corresponden al $26 \%$ de las publicaciones científicas, (Ver figura 1).

Figura 1. Número de artículos sobre RWH, relacionados con los tópicos de estudio.

\begin{tabular}{|c|c|c|c|c|c|c|}
\hline Superficies de cubierta & & & & & & \\
\hline $\begin{array}{l}\text { Calidad del agua } \\
\text { pluvial }\end{array}$ & & & & & & \\
\hline Usos del agua pluvial & & & & & & \\
\hline & 51 & 01 & 52 & 02 & 53 & 0 \\
\hline
\end{tabular}

Fuente: Elaboración propia a partir de base de datos de Esnob, Isi y Scopus.

La revisión contemplo el análisis de las publicaciones por región y países que han profundizado sobre las ventajas del almacenamiento y uso del agua pluvial: Asía ocupa el primer lugar, con 19 de los 50 artículos revisados, en segundo lugar está Norte américa, con 11 artículos publicados, en tercer lugar Europa con 10 publicaciones indexadas y en cuarto lugar Australia con 5 artículos publicados. Por países se 
encuentra que China es el país que mayor publicación a nivel mundial viene cosechando con 12 artículos de las 50 publicaciones revisadas, seguido de Estados Unidos con 7 artículos y en tercer lugar Francia y Colombia, que han publicado 3 artículos científicos en cada región a la que pertenece cada país, (Ver figura 2).

Figura 2. Número de artículos sobre RWH, publicados por país y año de publicación.

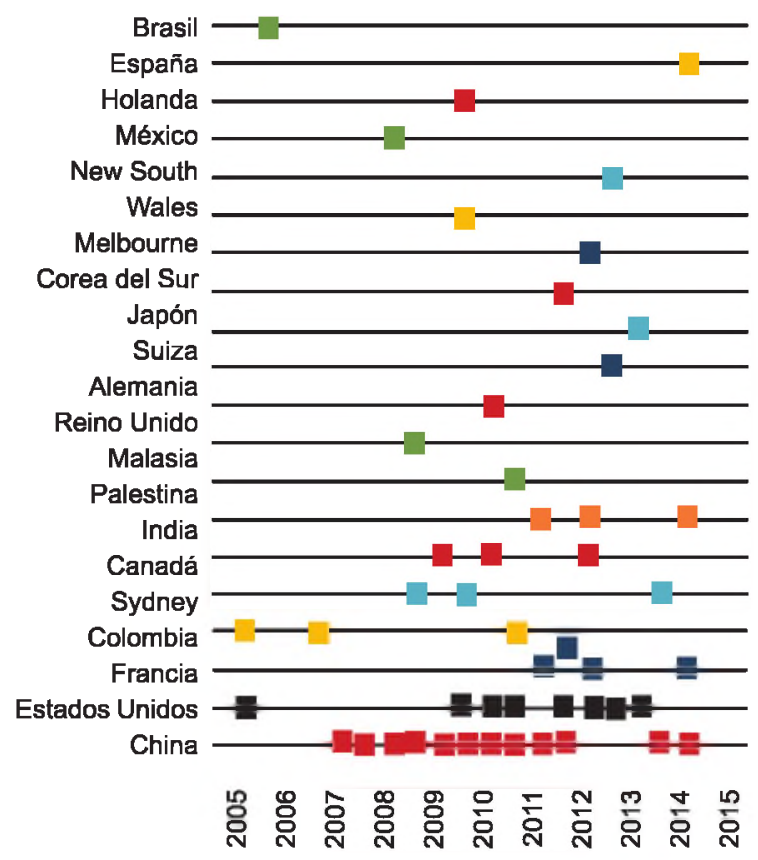

Fuente: Elaboración propia a partir de base de datos de Esnob, Isi y Scopus.

\section{MÉTODO}

El método planteado para el presente artículo se basa: i) indagación sobre el número de artículos que plantea los tópicos de interés; superficies de captación, variables que determinan la calidad del agua pluvial y por último calidad del agua pluvial, para ello se indago en 50 artículos publicados en revistas indexadas, ii) en segundo lugar la revisión se agrupó por número de artículos publicados por país y año de publicación centrándose la revisión en los últimos 10 años, la pertinencia y calidad del ar- tículo científico permitió profundizar en el área de estudio y iii) se generó una tabla (ver figura 3) que sintetiza los temas y número de artículos revisados en el presente artículo, asignando un código de color a los temas de la revisión y análisis del área de estudio.

\section{USOS DEL AGUA PLUVIAL}

El principal tópico que aborda la investigación son los usos del agua pluvial. Las publicaciones revisadas contemplan los siguientes variables a nivel mundial: consumo humano (Aladenola \& Adeboye, 2010), para potabilizar el agua pluvial para consumo humano requiere que la calidad de recogida y almacenamiento sea óptima, lo que hace que este uso este reducido a escala mundial, algunas ciudades de la India y China, la emplean bajo condiciones de higiene de mediana calidad. Los estudios sobre el uso del agua lluvia para la descarga de inodoros se concentra en edificios públicos con grandes ventajas en el ahorro y gestión del agua pluvial (Abbasi \& Abbasi, 2011), esta estrategia se ha implementado a mayor escala en Asia y Europa y a una menor escala en América, (Li et al, 2010), un avance significativo en términos de eficiencia, costos y beneficio ambiental lo describe el estudio realizado por los autores Chilton, et al (2000) en un sistema de recuperación de agua de lluvia aplicado en el Reino Unido, logrando una eficiencia de recolección del $57,4 \%$.

Solamente uno de los artículos revisados plantea el lavado de autos, sin embargo este uso se ha implementado de manera más espontánea en el mundo entero sin la implementación de tecnología adecuada ni para la captación, como tampoco para el almacenamiento. Un uso que se viene implementando de manera sistemática es el lavado y limpieza de fachadas (Blocken et al, 2013; Borrero et al, 2007), las publicaciones revisadas permiten apreciar los logros obtenidos y el óptimo beneficio en ahorro del recurso hídrico, muy utilizado en Norte y Sur América y como estrategia de sostenibilidad en Europa. Países como Suiza, Colombia y Estados Unidos 
LINNEA DE INVESTIGACIÓN: ARQUITECTURA, CIUDAD Y AMBIENTE

están entre los principales países que mayor aplicación dan al uso del agua pluvial para el lavado y mantenimiento de fachadas.

Otro importante uso que se hace del recurso pluvial es el riego de jardines (Woltersdorf et al, 2013 b), las regiones de Asia y Europa son pioneras en el riego de zonas verdes, manteniendo de jardines y en la implementación de sistemas por goteo que permiten una mayor eficacia en la gestión del agua (Gong et al, 2014), los sis- temas de almacenamiento y tratamiento son mínimos para garantizar la belleza de los jardines y huertas, Japón, Canadá y la India son un buen ejemplo de la implementación de sistemas de goteo permanente y en el mantenimiento de sus jardines domésticos a partir del agua lluvia (Biswas, \& Mandal, 2014). Por último encontramos que en menor medida el agua lluvia es un importante recurso en el abastecimiento de los sistemas de aire acondicionado, solo un artículo de la revisión aborda este tema.

Tabla 1. Usos del agua pluvial por países

\begin{tabular}{|c|c|c|c|c|c|c|}
\hline \multicolumn{7}{|c|}{ Usos del agua pluvial a escala global } \\
\hline Región/País & Inodoros & $\begin{array}{l}\text { Limpieza de } \\
\text { Fachadas }\end{array}$ & $\begin{array}{c}\text { Limpleza del } \\
\text { hogar }\end{array}$ & $\begin{array}{l}\text { Riego de } \\
\text { Jardínes }\end{array}$ & $\begin{array}{l}\text { Lavado de } \\
\text { autos }\end{array}$ & $\begin{array}{l}\text { Consumo } \\
\text { humano }\end{array}$ \\
\hline \multicolumn{7}{|l|}{ Canadá } \\
\hline \multicolumn{7}{|l|}{ Francia } \\
\hline \multicolumn{7}{|l|}{ Suiza } \\
\hline \multicolumn{7}{|l|}{ Colombia } \\
\hline \multicolumn{7}{|l|}{ Malasia } \\
\hline \multicolumn{7}{|l|}{ Inglaterra } \\
\hline \multicolumn{7}{|l|}{ Estados Unidos } \\
\hline \multicolumn{7}{|l|}{ Japón } \\
\hline \multicolumn{7}{|l|}{ China } \\
\hline \multicolumn{7}{|l|}{ México } \\
\hline \multicolumn{7}{|l|}{ Namibia } \\
\hline \multicolumn{7}{|l|}{ España } \\
\hline \multicolumn{7}{|l|}{ Palestina* $^{\star}$} \\
\hline \multicolumn{7}{|l|}{ Malasia* } \\
\hline India* & & & & & & \\
\hline *Baja calidad del & luvia p & isum & y & agua pota & & \\
\hline
\end{tabular}

Fuente: Elaboración propia a partir de la revisión de las referencias

\section{Gestión del recurso hídrico pluvial}

Considerando el buen manejo del recurso pluvial, algunos artículos de la revisión profundizan en la gestión del agua lluvia y la calidad de la misma, plantean incluso metodologías con gran éxito, como en París donde se concluye que es posible ahorrar hasta un $11 \%$ del agua potable corriente total a través del uso de RW
(Rainwater), específicamente para uso doméstico (Belmeziti et al, 2014). Otras publicaciones abordan métodos de gestión a partir de tres variables; el cálculo por demanda (per cápita), la capacidad de almacenamiento y la calidad del agua (Kim \& Furumai, 2012), en Japón se establece una clasificación de diferentes edificaciones de acuerdo con su función para el uso eficiente del recurso pluvial, en el siguiente or- 
den: residencial, oficinas, locales comerciales, restaurantes y edificios públicos.

En Australia las políticas ambientales se han incorporado a los proyectos de vivienda a partir del 2009, la viabilidad de la utilización de agua de lluvia en edificios de gran altura se viene implementando en cuatro ciudades australianas; Melbourne, Sydney, Perth y Darwin. El análisis de patrones climáticos y los distintos niveles de gestión de la demanda de agua se establecieron como parámetros para las dimensiones de almacenamiento; el consumo anual de agua del tanque; reducción tanto en el flujo de agua importada y disposición de aguas pluviales y derrames de agua de los tanques. (Zhang et al, 2009). Es interesante analizar cómo los patrones de precipitación determinan las dimensiones de los sistemas de almacenamiento; se encontró que para edificios de unidades múltiples, un mayor tamaño del tanque es más apropiado para maximizar el ahorro de agua, los resultados ya se hacen evidentes en edifi- cios de viviendas en tres ciudades de Australia: Sydney, Newcastle y Wollongong, (Eroksuz et al, 2010).

En la ciudad de Xiamen, China la aplicación del análisis de balance de agua diario sirve como estrategia para el diseño de la capacidad de almacenamiento y aprovechamiento de aguas pluviales, en zonas residenciales se establece bajo la siguiente ecuación; el balance hídrico relevante, y los métodos de cálculo de los principales factores en el balance de agua, incluyendo la recolección de agua de lluvia por escorrentía, el consumo de agua (per cápita), la evaporación y la infiltración (Wu et al, 2008). Los resultados muestran que la captación de agua de lluvia y de utilización de las superficies de techos urbanos, aceras, parques y espacios verdes pueden aumentar la recarga de suministro de los recursos hídricos y del agua subterránea (Xin-gao, et al, 2010). Cabe destacar el estudio realizado por "Lluviatl" en México, sobre la gestión integral del recurso hídrico (Pacheco, 2008).

Tabla 2. Gestión recurso hídrico pluvial por países

\begin{tabular}{|c|c|c|c|c|c|c|}
\hline \multicolumn{7}{|c|}{ Gestión recurso hídrico pluvial } \\
\hline $\begin{array}{l}\text { Regiónl } \\
\text { Pais }\end{array}$ & $\begin{array}{l}\text { Cálculo por } \\
\text { demanda }\end{array}$ & $\begin{array}{c}\text { Capacidad de } \\
\text { almacenamiento }\end{array}$ & $\begin{array}{l}\text { Patrones de } \\
\text { precipitación }\end{array}$ & $\begin{array}{c}\text { Análisis } \\
\text { de balance } \\
\text { diario }\end{array}$ & $\begin{array}{c}\text { Monitoreo; } \\
\text { techo patio, } \\
\text { carretera }\end{array}$ & $\begin{array}{l}\text { Cálculo y } \\
\text { Simulación }\end{array}$ \\
\hline Australia & \multicolumn{3}{|c|}{ Tamaño óptimo de los tanques } & & & \\
\hline $\begin{array}{l}\text { Reino } \\
\text { Unido* }\end{array}$ & & & & \multicolumn{2}{|c|}{ Gestión de aguas urbanas } & \\
\hline Suecia & & & & & & \\
\hline Francia & \multicolumn{2}{|c|}{ Ahorro del $11 \%$ de agua potable } & & & & \\
\hline $\begin{array}{l}\text { Estados } \\
\text { Unidos }\end{array}$ & \multicolumn{4}{|c|}{ Análisis comparativos por regiones } & & \\
\hline \multicolumn{7}{|l|}{ China } \\
\hline \multicolumn{7}{|l|}{ Japón } \\
\hline India & Análisis de vic & a util de los tanque: & s a 25 años & & & \\
\hline
\end{tabular}

Fuente: Elaboración propia a partir de la revisión de las referencias 
LIINEA DE INVESTIGACIÓN: ARQUITECTURA, CIUDAD Y AMBIENTE

\section{VARIABLES QUE DETERMINAN LA CALIDAD DEL AGUA PLUVIAL}

Existe amplia información relacionada con la calidad del recurso pluvial, las publicaciones analizan aspectos relacionados con la calidad del agua lluvia almacenada, que incluyen la medición de $\mathrm{pH}$, alcalinidad, dureza, sólidos totales disueltos (TDS), hierro, cloruro, nitrato, y la turbidez, utilizando diversos métodos de análisis. Sin embargo autores como Ahmed, et al (2011), señalan las consecuencias sobre este estudio que están asociadas a monitorear la calidad microbiana de agua lluvia y da una idea de los riesgos para la salud y los posibles brotes de enfermedades asociadas con el consumo de agua lluvia almacenada sin tratar, específicamente con bacterias fecales. En esta línea también Despins, et al (2009), estudian otras variables que determinan la calidad del agua lluvia, como son: color, coliformes totales y fecales, carbono orgánico total, nitrógeno total y absortancia, incluso los parámetros adicionales; incluyendo hidrocarburos aromáticos, policíclicos y metales totales son evaluadas por métodos de recolección de datos que se realizaron a partir de análisis estadísticos para investigar los factores que influyen en la calidad del agua de lluvia, tanto en zonas rurales, como urbanas (Kus et al, 2011)

Dentro de la revisión otros autores señalan que la calidad del agua lluvia depende del uso y disposición final del recurso, análisis posteriores a la recolección de agua lluvia, arrojan variantes dependiendo la época del año, parámetros microbiológicos fluctuaron durante el estudio, con los más altos niveles de contaminación microbiológica observado en escorrentías de techos que recogían el agua durante un tiempo determinado (Vialle et al, 2012; Montréjaud-Vignoles et al, 2012), los autores señalan en general, que el agua de lluvia recogida tiene una relativa buena calidad fisicoquímica pero variable, sin embargo no cumple con estándares para el consumo humano. Los estudios e investigaciones sobre la calidad del agua lluvia se concentran en Europa, particularmente en Francia (De Gouvello et al, 2012), estos autores plantean en los artículos publicados estrategias y métodos de evaluación y análisis del agua lluvia y su potencial uso como alternativa a la crisis mundial del agua.

Otros estudios realizados en Canadá y Estados Unidos (DeBusk et al, 2013), arrojan como resultados; que, mientras que la calidad varía bajo condiciones ambientales, el agua de lluvia puede ser de alta calidad a través de la selección de materiales de captación y almacenamiento apropiados y la aplicación de tratamiento post-captación (Despins et al, 2009 b). En esta misma línea un estudio demostró que las condiciones climáticas; intensidad de lluvias y variaciones de temperatura afectan la calidad microbiológica del agua almacenada y es más propensa a la contaminación de indicadores fecales y el aumento de la detección de agentes patógenos después de los incidentes de lluvias fuertes (Schets, et al, 2010).

En los países con grandes demandas de agua y crisis del recurso han optado por la recolección del agua lluvia como alternativa, Palestina es uno de los países que han implementado esta práctica, considerando esta premisa la revisión de las publicaciones encontró que las cuantificaciones que miden la calidad del agua se fundan en los siguientes parámetros fisicoquímicos: temperatura, $\mathrm{pH}$, conductividad eléctrica, salinidad, sólidos disueltos totales, turbidez, nitrato, cobre y plomo. Con pocas excepciones, todos estos parámetros estaban dentro de los valores de referencia de la Organización Mundial de la Salud OMS, (Daoud et al, 2011).

\section{Calidad del almacenamiento: sistemas de captación y reciclaje del agua pluvial}

Otro aspecto que concierne a la calidad del agua lluvia, se concentra en los sistemas de almacenamiento, con fines específicos, algunos ya mencionados anteriormente; jardinería, limpieza de elementos sólidos y con fines de mantenimiento; los sistemas de almacenamiento para aplicaciones de menor escala con tan- 
Tabla 3. Variables que determinan la calidad del agua pluvial por países

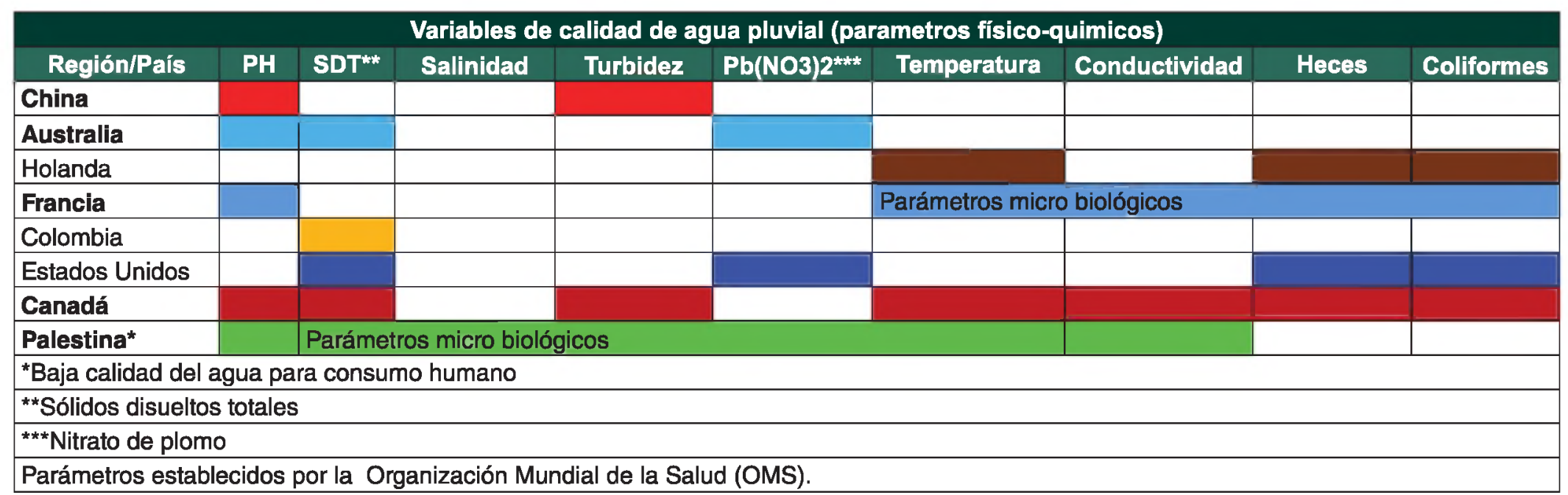

Fuente: Elaboración propia a partir de la revisión de las referencias 
defines tres tipos de cubierta: tejas de asfalto y fibra de vidrio, metal galvanizado y baldosas de hormigón, dan como resultado que las superficies metálicas, garantizan una mejor calidad del agua lluvia captada, sin embargo se requieren estudios más profundos para determinar su potencial uso dado que las evaluaciones arrojan residuos de metales pesados (Magyar et al, 2014). Sobre esta misma línea, también otros autores entre ellos Cupido, et al, (2012) realizan análisis en superficies de membranas de color blanco y muestran interesantes resultados; señala el estudio que la favorabilidad de las membranas, supera a las demás superficies, garantizando mayor calidad del agua Iluvia, para posible aplicaciones y usos del recurso en edificios públicos, esta aplicación se viene realizando con gran éxito en Canadá. Un importante estudio desarrollado en Colombia, muestra como el agua lluvia de escorrentía sobre tejados de fibro-cemento, se podría adaptar para ser utilizada como fuente alternativa para satisfacer algunos usos domésticos (Torres et al, 2011).

\section{Superficies de techos verdes}

Los techos verdes en comparación con otras superficies presentan grandes beneficios ambientales, entre ellas mitigar las crecidas en zonas urbanas, uno de los artículos más interesantes plantea algunos sistemas de drenaje a diferentes profundidades; entre $5 \mathrm{~cm}$. y $14 \mathrm{~cm}$, para observar el comportamiento de las plantas, sus raíces y como afecta el flujo de las aguas pluviales, los resultados mostraron que el aumento de la profundidad del suelo "techo verde" mejora la retención de agua y tiempo de retraso de la escorrentía (Buccola \& Spolek, 2011). Otros artículos de la revisión señalan; que los techos verdes también reducen la turbidez, el valor químico del oxígeno y neutraliza la lluvia ácida para estabilizar el pH (Gong et al, 2014). En esta misma línea los autores Morgan, et al, (2012), indican que los sistemas de techo verde reducen significativamente el escurrimiento del agua de lluvia y el diseño de los sistemas de retención, la profundidad de los sustratos de crecimiento y la presencia de plantas mejoran la retención de aguas pluviales.

\section{Uso y tecnología del agua pluvial}

Los sistemas de tratamiento han hecho posible que la calidad del agua pluvial pueda convertirse en una alternativa confiable, eficiente y sostenible, las publicaciones revisadas en este tema apuntan a implementar tecnología de punta, es así como un estudio realizado en Australia muestra las bondades del carbón activado granular (GAC), que funciona como filtro de adsorción, el refuerzo GAC elimina los contaminantes a través de un mecanismo de adsorción (Kus et al, 2011). Otro importante aporte lo realizan los autores Lstiburek, et al (2005), quienes crearon un sistema de recolección de aguas lluvias a través de las fachadas con un sistema de protección multicapas. En el contexto urbano, el aprovechamiento de aguas pluviales en la ciudad tiene una gran importancia para el desarrollo sostenible de la sociedad, el medio ambiente y la economía (Wang, et al 2007; Xin-gao et al, 2010).

En esta línea de pensamiento los investigadores del Reino Unido, Ward, Memon, \& Butler (2012) plantean dos interesantes sistemas de captación; se compara el desempeño real con el rendimiento estimado basado en dos métodos recomendados por el (British Standards Institute) en primer lugar: un sistema Intermedio (cálculos sencillos) y el segundo, un sistema Detallado (basado en la simulación) denominado Enfoques. "Los resultados destacan que el ahorro de agua promedio de eficiencia medido (cantidad de agua de la red guardada) del sistema de captación de aguas pluviales en la consulta fue del $87 \%$ a través de un período de 8 meses, debido al sistema de gran tamaño para el nivel de ocupación real".

Por último un interesante estudio analiza los sistemas de captación que se han generado en la historia de la humanidad para suplir las necesidades de abastecimiento del recurso hídrico a nivel mundial: "los sistemas de aprovechamiento de agua lluvia sólo se implementan cuando no existe una red de acueducto, el suministro es deficiente, la calidad del agua es muy baja o los costos del agua potable son muy altos"(Suárez, et al, 2006). 
LIINEA DE INVESTIGACIÓN: ARQUITECTURA, CIUDAD Y AMBIENTE

\section{CONCLUSIONES}

Actualmente existe en el mundo una vasta producción científica sobre el tema de calidad del agua lluvia ( $\mathrm{RWH}$, siglas en inglés). Sin embargo los énfasis temáticos y tópicos de investigación se centran en la disposición final del recurso pluvial y las variables para determinar la calidad del agua, desde su captación hasta su disposición en tanques de almacenamiento. Los estudios relacionados sobre los usos también son amplios y bien detallados, las publicaciones determinan muy bien las condiciones de calidad que debe tener el agua recolectada a partir de análisis microbiológicos hasta serios estudios estadísticos de patrones de precipitación, demográficos y tipo de edificación. Sin embargo las publicaciones sobre el tema de superficies de cubierta son escasas, algunos artículos mencionan algún tipo de material, otras publicaciones la eficiencia en cuanto a carga de escorrentía, tal como lo mencionan los artículos relacionados con techos verdes, pero no existen estudios que profundicen sobre materiales que garanticen la sostenibilidad integral en todo el proceso de captación, disposición y destino final del recurso pluvial. (Ver figura 3 ).

Tabla 5. Uso eficiente del agua lluvia; ventajas y desventajas

\begin{tabular}{|c|c|c|}
\hline \multicolumn{3}{|c|}{ Uso eficiente del recurso pluvial } \\
\hline Tópicos & Ventajas & Desventajas \\
\hline \multirow{3}{*}{ 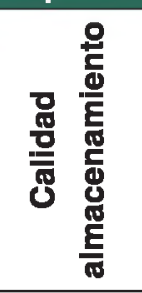 } & $\begin{array}{l}\text { - Beneficios en términos tecnológicos, } \\
\text { económicos, ambientales y sociales. }\end{array}$ & \multirow{2}{*}{$\begin{array}{l}\text { - No existen políticas de recogida y almacena- } \\
\text { miento de aguas pluviales. *Aumenta la recarga } \\
\text { de suministro de los recursos hídricos y las } \\
\text { aguas subterráneas. }\end{array}$} \\
\hline & $\begin{array}{l}\text { - Aliviar el conflicto entre la oferta y de- } \\
\text { manda de agua en las ciudades. } \\
\end{array}$ & \\
\hline & - Fuente alternativa de agua & $\begin{array}{l}\text { - Minimizar el sobre-dimensionamiento de los } \\
\text { tanques de almacenamiento. }\end{array}$ \\
\hline \multirow{4}{*}{ 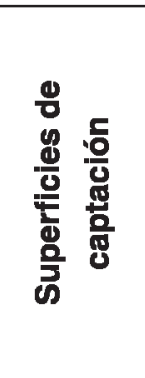 } & $\begin{array}{l}\text { - Utilización sostenible de los recursos } \\
\text { hídricos. }\end{array}$ & \multirow{2}{*}{$\begin{array}{l}\text { - El agua de lluvia almacenada por mucho tiempo } \\
\text { en diferentes reservorios es propenso a la con- } \\
\text { taminación con heces y patógenos humanos. }\end{array}$} \\
\hline & - La recolección de agua de lluvia de los & \\
\hline & $\begin{array}{l}\text { techos, su almacenamiento y posterior } \\
\text { uso de inodoro puede ahorrar } 42 \mathrm{~m} 3 \\
\text { de agua potable al año. }\end{array}$ & \multirow{2}{*}{$\begin{array}{l}\text { - Factores que afectan la calidad del agua lluvia } \\
\text { almacenada; material de cubierta, la tempera- } \\
\text { tura del sitio, la frecuencia de uso del tanque, y } \\
\text { uso de la tierra circundante. }\end{array}$} \\
\hline & $\begin{array}{l}\text { - Las cubiertas vegetales evitan daños } \\
\text { por escorrentía con fuertes lluvias }\end{array}$ & \\
\hline \multirow{4}{*}{ 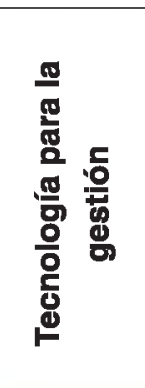 } & $\begin{array}{l}\text { - Beneficios económicos, sociales y eco- } \\
\text { lógicos al medio ambiente. } \\
\text { - Ahorro de agua y de costos. } \\
\end{array}$ & $\begin{array}{l}\text { - Los costos de inversión en materiales plantean } \\
\text { un problema de financiación. }\end{array}$ \\
\hline & $\begin{array}{l}\text { - Aparatos de bajo consumo de agua } \\
\text { estándar. }\end{array}$ & \multirow{2}{*}{$\begin{array}{l}\text { - Mejorar los métodos de desinfección y la gestión } \\
\text { desde la captación de agua para evitar la conta- } \\
\text { minación en los sistemas de almacenaje. }\end{array}$} \\
\hline & \multirow{2}{*}{$\begin{array}{l}\text { - Reducción de la escorrentía de aguas } \\
\text { pluviales; aplicación en cuencas de } \\
\text { drenaje residencial }\end{array}$} & \\
\hline & & - Los costos del agua potable son muy altos \\
\hline
\end{tabular}

Fuente: Elaboración propia a partir de la revisión de las referencias 
Figura 3. Número de artículos revisados RWH, publicados por tema, región, país y año de publicación.

\begin{tabular}{|c|c|c|c|c|c|c|c|}
\hline \multirow{2}{*}{$\begin{array}{l}\text { Rovisi } \\
\text { Articulo }\end{array}$} & \multicolumn{4}{|c|}{ Tópicos o temas de análisis } & \multicolumn{3}{|c|}{ Publicación indexada } \\
\hline & Uso & Calidad & Superficie & Región & País & Tema & Año \\
\hline 1 & & $\mathbf{x}$ & & Asia & India & Estrategias de gestión & 2011 \\
\hline 2 & & $\bar{x}$ & $\bar{x}$ & Norte América & E.U & Material y agua pluvial & 2011 \\
\hline 3 & & $\mathbf{x}$ & & Norte América & E.U & Calidad del agua en superticies & 2010 \\
\hline 4 & $\mathbf{x}$ & & & Narte América & Ganadá & Fotencial agua |luvia/uso doméstico & 2010 \\
\hline 5 & $x$ & $x$ & & Asia & India & Uso doméstico del agua pluvial/calidad & 2014 \\
\hline 6 & $\mathbf{x}$ & & & Europa & Francia & Recogida agua pluvial en Paris & 2014 \\
\hline 7 & $x$ & & & Europa & Suiza & Lavado de tachadas & 2013 \\
\hline 8 & $\bar{x}$ & & & Sur América & Colambia & Lavado de fachadas & 2007 \\
\hline $\mathbf{9}$ & & & $\mathbf{x}$ & Norte América & E.U & Techos verdes/gestión recursa hídrica & 2010 \\
\hline 10 & $\mathbf{x}$ & & & Asia & Malasia & Reciclado de agua pluvial & 2009 \\
\hline 11 & $x$ & & $x$ & Europa & UK & Reciclado de agua pluvial, para inodoro & 2010 \\
\hline 12 & & $\bar{x}$ & $\bar{x}$ & Norte América & Canadá & Superficies de membranas (Betún, PVC, TPO) & 2012 \\
\hline 13 & & $\mathrm{x}$ & & Asia & Palestina & Calidad del agua pluvial almacenada & 2011 \\
\hline 14 & & $\mathbf{x}$ & & Europa & Francia & Evaluación calidad del agua & 2012 \\
\hline 15 & $\mathrm{x}$ & & & Norte América & E.U & Caracterización agua pluvial en E. U & 2013 \\
\hline 16 & & $\mathbf{x}$ & & Norte América & Canadá & Evaluación calidad del agua & 2009 \\
\hline 17 & $\mathbf{x}$ & & & Australia & Sydney & Almacenamiento de agua pluvial & 2010 \\
\hline 98 & & $\mathbf{x}$ & $\mathbf{x}$ & Asia & China & Calidad de agua lluvia en techos verdes & 2014 \\
\hline 19 & & $\mathbf{x}$ & $\mathbf{x}$ & Asia & China & Almacenamiento de agua pluvial & 2014 \\
\hline 20 & $\mathbf{x}$ & & $\mathbf{x}$ & Asia & China & Almacenamiento de agua pluvial & 2008 \\
\hline 21 & & & $\mathbf{x}$ & Asia & India & Costo almacenamiento agua lluvia & 2012 \\
\hline 22 & $\mathbf{x}$ & & & Australia & Melbourne & Dimensionamiento óptimo tanques de reserva & 2009 \\
\hline 23 & $x$ & & & Asia & Japón & Evaluación por tipo de consirucción & 2012 \\
\hline 24 & & $\mathbf{x}$ & & Australia & New South Wales & Calidad del ague en los tanques & 2013 \\
\hline 25 & & $x$ & $x$ & Asia & Gorea del Sur & Galidad del agua en superficies de techos & 2012 \\
\hline 26 & $\mathbf{x}$ & & & Asia & China & Agua lluvia para el inodoro & 2010 \\
\hline 27 & $\mathbf{x}$ & & & Norte América & E.U & Control de humedad en las fachadas & 2005 \\
\hline 28 & $x$ & & & Asia & China & Jardín y gestión del agua lluvia & 2008 \\
\hline 29 & & $\mathbf{x}$ & $x$ & Australia & Sydney & $\begin{array}{l}\text { Contaminación del agua por superficies de } \\
\text { cubierta }\end{array}$ & 2014 \\
\hline 30 & & $\mathbf{x}$ & $\mathrm{x}$ & Norte América & E.U & Calidad del agua en techos verdes & 2012 \\
\hline 31 & $\mathbf{x}$ & & & Centro América & México & Gestión del agua y consumo sostenible & 2008 \\
\hline 32 & $x$ & & & Sur América & Colambia & Uso del agua lluvia, múltiples usos & 2004 \\
\hline 33 & $\mathbf{x}$ & $\mathbf{x}$ & & Eurapa & Halanda & Evaluación calidad del agua pluvial & 2010 \\
\hline 34 & $\mathbf{x}$ & & & Norte América & E.U & $\begin{array}{l}\text { Gestión y recolección del agua pluvial en } \\
\text { residencias }\end{array}$ & 2013 \\
\hline 35 & & $\mathbf{x}$ & & Norte América & E.U & Calidad del agua y sistemas de captación & 2012 \\
\hline 36 & $\mathbf{x}$ & & & Sur América & Brasil & Sistemas de aprovechamiento de aguas lluvias & 2006 \\
\hline 37 & $x$ & $x$ & & Sur América & Colombia & $\begin{array}{l}\text { Calidad de la escorrentía de agua pluvial en } \\
\text { Bogotá }\end{array}$ & 2011 \\
\hline 38 & & $x$ & $x$ & Europa & España & $\begin{array}{l}\text { Sistemas de recolección aguas pluviales, } \\
\text { Earcelona }\end{array}$ & 2014 \\
\hline 39 & & $\mathbf{x}$ & $\mathrm{x}$ & Europa & Francia & $\begin{array}{l}\text { Calidad del agua pluvial en una vivienda en } \\
\text { Francia }\end{array}$ & 2012 \\
\hline 40 & & $\mathbf{x}$ & $\mathbf{x}$ & Europa & Francia & Galldad del agua pluvial en los techos & 2011 \\
\hline 41 & $x$ & $x$ & & Europa & Suecia & $\begin{array}{l}\text { Recolección de agua lluvia para el suministro } \\
\text { de agua doméstica }\end{array}$ & 2008 \\
\hline 42 & $\mathbf{x}$ & $\mathbf{x}$ & & Asia & China & Calidad del agua lluvia, uso y recolección & 2008 \\
\hline 43 & $x$ & & & Asia & China & Uso y tecnologla del agua lluvia & 2007 \\
\hline 44 & $\mathbf{x}$ & & & Asia & China & Uso y tecnologla del agua lluvia en edificios & 2010 \\
\hline 45 & $\mathbf{x}$ & $\mathbf{x}$ & & Asia & China & Galidad del agua lluvia en la escorrentía & 2009 \\
\hline 46 & $\mathbf{x}$ & & & Europa & Alemania & Agua lluvia para jardinería & 2013 \\
\hline 47 & $x$ & & & Asia & China & $\begin{array}{l}\text { Almacenamiento de agua lluvia en zonas } \\
\text { residenciales }\end{array}$ & 2008 \\
\hline 48 & $\mathbf{x}$ & & & Asia & China & Fotencial del agua lluvia & 2009 \\
\hline 49 & & $\mathbf{x}$ & & Asla & China & $\begin{array}{l}\text { Galidad del agua lluvia, en poblaciones en } \\
\text { China }\end{array}$ & 2009 \\
\hline 50 & $x$ & $x$ & & Australia & Cities & Uso del agua lluvia en edificios en altura & 2009 \\
\hline
\end{tabular}

Fuente: Elaboración propia a partir de base de datos de Esnob, Isi y Scopus. 


\section{REFERENCIAS}

Abbasi, T., \& Abbasi, S. A. 2011. Sources of pollution in rooftop rainwater harvesting systems and their control. Critical Reviews in Environmental Science and Technology, 41(23): 2097-2167.

Afshar, B. R., Kinney, K., Barrett, M. E., \& Kirisits, M. J. 2010. Effect of roof material on water quality for rainwater harvesting systems. Texas Water Development Board.

Ahmed, W., Gardner, T., \& Toze, S. 2011. Microbiological quality of roof-harvested rainwater and health risks: a review. Journal of environmental quality, 40 (1), 13-21.

Aladenola, O. O., \& Adeboye, O. B. 2010. Assessing the potential for rainwater harvesting. Water Resources Management, 24(10): 2129-2137.

Biswas, B. K., \& Mandal, B. H. 2014. Construction and evaluation of rainwater harvesting system for domestic use in a remote and rural area of Khulna, Bangladesh. International Scholarly Research Notices, 2014

Belmeziti, A., Coutard, O., \& de Gouvello, B. 2014. How much drinking water can be saved by using rainwater harvesting on a large urban area? Application to Paris agglomeration. Water Science \& Technology, 70 (11), 1782-1788.
Blocken, B., D. Derome, and J. Carmeliet. 2013. Rainwater runoff from building facades: A review. Building and Environment 60: 339-61.

Borrero, J. A. L., Abello, A. E. T., Pinilla, M. C. C., Castro, L. D., \& Robayo, J. I. E. 2007. Aprovechamiento del agua lluvia para riego y para el lavado de zonas duras y fachadas en el campus de la Pontificia Universidad Javeriana (Bogotá). Ingeniería y Universidad, 11 (2): 193-202.

Buccola, N., \& Spolek, G. 2011. A pilot-scale evaluation of greenroof runoff retention, detention, and quality. Water, Air, \& Soil Pollution, 216 (1-4): 83-92.

Che-Ani, Al, N. Shaari, A. Sairi, MFM Zain, and MM Tahir. 2009. Rainwater harvesting as an alternative water supply in the future. European Journal of Scientific Research 34 (1): 13240.

Chilton, JC, GG Maidment, D. Marriott, A. Francis, and G. Tobias. 2000. F. Urban Water 1 (4): 345-54.

Cupido, A., Baetz, B., Guo, Y., \& Robertson, A. 2012. An evaluation of rainwater runoff quality from selected white roof membranes. Water Quality Research Journal of Canada, 47 (1): 66-79.

Daoud, A., Swaileh, K., Hussein, R., \& Matani, M. 2011. Quality assessment of roof-har- vested rainwater in the West Bank, Palestinian Authority. Journal of water and health, 9 (3): 525-533.

De Gouvello, B., Nguyen - Deroche, N., Lucas, F., \& Gromaire, M. C. 2012. A methodological strategy to analyze and improve the French $\mathrm{RWH}$ regulation on quality topic. Water Science and Technology, 67 (5): 1043-1050.

DeBusk, K. M., Hunt, W. F., \& Wright, J. D. 2013. Characterizing rainwater harvesting performance and demonstrating storm water management benefits in the humid southeast.

Despins, C., Farahbakhsh, K., \& Leidl, C. 2009. Assessment of rainwater quality from rainwater harvesting systems in Ontario, Canada. Aqua, 58 (2): 117.

Eroksuz, Erhan, and Ataur Rahman. 2010. Rainwater tanks in multi-unit buildings: A case study for three Australian cities. Resources, Conservation and Recycling 54 (12): 1449-52.

Gong, K., Wu, Q., Peng, S., Zhao, X., \& Wang, X. 2014. Research on the characteristics of the water quality of rainwater runoff from green roofs. Water Science \& Technology, 70 (7): 1205-1210.

Guo, Yiping, and Brian W. Baetz. 2007. Sizing of rainwater storage units for green building applications. Journal of 
Hydrologic Engineering 12 (2): 197-205.

Guoqing, Pan, Che Wu, Li Junqi, Li Haiyan, He Jianping, and Wang Hongling. 2008. Study on the volume optimization for rainwater collection by storage tank. Water and Wastewater Engineering 34 (12): 42-7.

Kumari, R., \& Sherring, A. 2012. Planning and cost estimation of roof rainwater harvesting structure" A case study". International Journal of Agriculture Environment \& Biotechnology, 5 (3): 225-232.

Khastagir, A., \& Jayasuriya, N. 2010. Optimal sizing of rain water tanks for domestic water conservation. Journal of Hydrology, 381 (3): 181-188.

Kim, J., \& Furumai, H. 2012. Assessment of rainwater availability by building type and water use through GIS-based scenario analysis. Water resources management, 26 (6): 1499-1511.

Kus, B., Jaya K., Saravanamuth V., and Hokyong S., 2011. Water quality in rainwater tanks in rural and metropolitan areas of New South Wales, Australia.

Lee, J. Y., Bak, G., \& Han, M. 2012. Quality of roof-harvested rainwater-Comparison of different roofing materials. Environmental Pollution, 162, 422-429.

$\mathrm{Li}$, TT, JR Liu, Dong $\mathrm{Li}$, and ZJ Liu. 2010. Case analysis of using roof rainwater as toilet flushing water. China Water \& Wastewater 26 (16): 122-5.

Lstiburek, Joseph William, P. Eng, and MA Westford. 2005. Rainwater management performance of newly constructed residential building enclosures during August anFd September.

Luo, Hong-mei, Wu CHE, Jun-qi LI, Hong-ling WANG, Guang-hui MENG, and Jianping HE. 2008. Application of rainwater garden to storm and flood control and utilization. China Water and Wastewater 24 (6): 48.

Magyar, M. I., Ladson, A. R., Diaper, C., \& Mitchell, V. G. 2014. Influence of roofing materials and lead flashing on rainwater tank contamination by metals. Australian Journal of Water Resources, 18(1): 71.

Morgan, S., Celik, S., \& Retzlaff, W. 2012. Green roof storm-water runoff quantity and quality. Journal of Environmental Engineering, 139(4): 471478.

Pacheco, M. M. 2008. Avances en la Gestión Integral del Agua Lluvia (GIALL): contribuciones al consumo sostenible del agua, el caso de" Lluviatl" en México. Revista Internacional de sostenibilidad, tecnologia y humanismo, (3): 39-57.

Sánchez, L. D., \& Caicedo, E. Y. 2004. Uso del Agua Lluvia en la Bocana Buenaventura. Universidad del Valle. Usos
Múltiples del Agua: para la Vida y el Desarrollo Sostenible. Cartagena de Indias, CINARA: 1-9.

Schets, F., Italiaander, R., Van Den Berg, H. H. J. L., \& de Roda Husman, A. 2010. Rainwater harvesting: quality assessment and utilization in The Netherlands. Journal of water and health, 8(2): 224-235.

Steffen, J., Jensen, M., Pomeroy, C. A., \& Burian, S. J. (2013). Water supply and stormwater management benefits of residential rainwater harvesting in US cities. JAWRA Journal of the American Water Resources Association, 49 (4), 810-824.

Stump, B., McBroom, M., \& Darville, R. 2012. Demographics, practices and water quality from domestic potable rainwater harvesting systems. Journal of Water Supply: Research and TechnologyAQUA, 61(5): 261-271.

Suárez, J., Garcia, M., \& Mosquera, R. 2006. Historia de los sistemas de aprovechamiento de agua lluvia. VI SEREA. Seminario lberoamericano sobre Sistemas de Abastecimiento Urbano de Agua João Pessoa (Brasil), 5.

Torres, A., Méndez-Fajardo, S., López-Kleine, L., Marín, V., González, J. A., Suárez, J. C. \& Ruiz, A. 2011. Evaluación preliminar de la calidad de la escorrentía pluvial sobre tejados para su posible aprovechamiento en zonas periurbanas 
LINNEA DE INVESTIGACIÓN: ARQUITECTURA, CIUDAD Y AMBIENTE

de Bogotá. Revista UDCA Act. \&Div. Cient, 14 (1):127-135.

Vargas-Parra, M., Rovira, M., Gabarrell, X., \& Villalba, G. 2014. Cost-effective rainwater harvesting system in the metropolitan area of Barcelona. Journal of Water Supply: Research and Technology-AQUA, 63 (7): 586-595.

Vialle, C., Sablayrolles, C., Lovera, M., Huau, M., Jacob, S., \& Montréjaud-Vignoles, M. 2012. Water quality monitoring and hydraulic evaluation of a household roof runoff harvesting system in France. Water resources management, 26 (8): 2233-2241.

Vialle, C., Sablayrolles, C., Lovera, M., Jacob, S., Huau, M. C., \& Montréjaud-Vignoles, $M$. 2011. Monitoring of water quality from roof runoff: Interpretation using multivariate analysis. Water Research, 45 (12): 37653775.

Villarreal, Edgar L., and Andrew Dixon. 2005. Analysis of a rainwater collection system for domestic water supply in ringdansen, norrköping, swe- den. Building and Environment 40 (9): 1174-84.

WANG, Guang-zhou, and Jian-en GAO. 2008. Problems of rainwater quality in rainfall collection and usage and its countermeasure [J]. Journal of Irrigation and Drainage 4: 034.

Wang, Hui-zhen, and JQ Wu. 2007. Technology and analysis of rain water utilization in city. Industrial Water \& Wastewater 38 (1): 9.

Ward, S., FA Memon, and D. Butler. 2012. Performance of a large building rainwater harvesting system. Water Research 46 (16): 5127-34.

WEI, Sa, Yong-chen GUO, and Jun WANG. 2009. The quality monitoring and influencing factors of urban area runoff [J]. Journal of Hebei Normal University (Natural Science Edition) 1: 032.

Woltersdorf, L., Jokisch, A., \& Kluge, T. (2013). Benefits of rainwater harvesting for gardening and implications for future policy in Namibia.
WU, Peng, Shao-hua CHEN, Chang-zhou YAN, and Kai-song ZHANG. 2008. Design of storage volumes for rainwater utilization in urban residential areas based on daily water balance [J]. China Water \& Wastewater 16: 014.

Xin-gao, LU, Pang Qingjiang, and Deng Aili. 2010. Potential, bene-fit analysis and utilization mode of urban rainwater resources. Journal of Economics of Water Resources 1: 1-4.

ZHANG, Guozhen, Chunsheng $H E$, and Fuping WU. 2009. Evaluation system of water quality for source rainwater in villeages and towns of Northwestern China [J]. Journal of Arid Land Resources and Environment 11: 020.

Zhang, Yan, Donghui Chen, Liang Chen, and Stephanie Ashbolt. 2009. Potential for rainwateruse in high-rise buildings in Australian cities. Journal of Environmental Management 91 (1): $222-6$ 